\title{
Anti-Tuberculosis Activity of Pediococcus acidilactici Isolated from Young Radish Kimchi against Mycobacterium tuberculosis
}

Youjin Yoon ${ }^{1 \dagger}$, Hoonhee $\mathrm{Se}^{2 \dagger}$, Sukyung Kim ${ }^{2}$, Youngkyoung Lee', MD Abdur Rahim ${ }^{1}$, Saebim Lee ${ }^{2}$, and Ho-Yeon Song ${ }^{1,2 *}$

${ }^{1}$ Department of Microbiology and Immunology, School of Medicine, Soonchunhyang University, Cheonan 31151, Republic of Korea

${ }^{2}$ Probiotics Microbiome Convergence Center, Soonchunhyang University, Asan 31538, Republic of Korea

Tuberculosis is a highly contagious disease caused by Mycobacterium tuberculosis. It affects about 10 million people each year and is still one of the leading causes of death worldwide. About 2 to 3 billion people (equivalent to 1 in 3 people in the world) are infected with latent tuberculosis. Moreover, as the number of multidrug-resistant, extensively drug-resistant, and totally drug-resistant strains of M. tuberculosis continues to increase, there is an urgent need to develop new anti-tuberculosis drugs that are different from existing drugs to combat antibiotic-resistant $M$. tuberculosis. Against this background, we aimed to develop new anti-tuberculosis drugs using probiotics. Here, we report the anti-tuberculosis effect of Pediococcus acidilactici PMC202 isolated from young radish kimchi, a traditional Korean fermented food. Under coculture conditions, PMC202 inhibited the growth of M. tuberculosis. In addition, PMC202 inhibited the growth of drug-sensitive and -resistant M. tuberculosis-infected macrophages at a concentration that did not show cytotoxicity and showed a synergistic effect with isoniazid. In a 2-week, repeated oral administration toxicity study using mice, PMC202 did not cause weight change or specific clinical symptoms. Furthermore, the results of $16 S$ rRNA-based metagenomics analysis confirmed that dysbiosis was not induced in bronchoalveolar lavage fluid after oral administration of PMC202. The anti-tuberculosis effect of PMC202 was found to be related to the reduction of nitric oxide. Our findings indicate that PMC202 could be used as an anti-tuberculosis drug candidate with the potential to replace current chemicalbased drugs. However, more extensive toxicity, mechanism of action, and animal efficacy studies with clinical trials are needed.

Keywords: Mycobacterium tuberculosis, Pediococcus acidilactici, probiotics, anti-tuberculosis effect, microbiome

\section{Introduction}

Mycobacterium tuberculosis, the causative agent of human tuberculosis, is one of the most prevalent human pathogens. It infects a quarter of the world's population by developing sophisticated mechanisms to bypass the host's innate and adaptive immune defenses [1]. Tuberculosis remains the leading cause of death from infectious diseases among adults worldwide [2]. According to a WHO report, in 2019, about 10 million people were infected with tuberculosis and about 1.4 million of them died from the disease [3]. Moreover, the pathogenic organism $M$. tuberculosis continues to evolve and increase its resistance to antagonists, leading to the development of multidrug-resistant (MDR) and extensively drug-resistant (XDR) M. tuberculosis strains, and ultimately, unmanageable totally drug-resistant (TDR) $M$. tuberculosis strains with even further developed resistance mechanisms [4]. It has been estimated that $3.7 \%$ of new cases of tuberculosis infection worldwide and $20 \%$ of previously treated cases are MDR tuberculosis [5], with a treatment success rate of only 54\% [6]. As such, the development of a new anti-tuberculosis drug to solve the evolving tuberculosis problem is urgently needed.

Probiotics are defined as "live microorganisms which when administered in adequate amounts confer a health benefit on the host" that can be applied as single or multiple strains in a live or dead form in combination with prebiotics [7]. Probiotics can significantly affect the functions of the mucosal and systemic immune system through activation of several immune mechanisms. They are effective in controlling various diseases, including irritable bowel disease, allergies, diabetes, and cancer [8]. In addition to these diseases, probiotics have been extensively studied for controlling infectious diseases and pathogens [9] such as Helicobacter pylori [10], salmonellosis [11], candidal vulvovaginitis [12], urinary tract infection [13], Clostridium difficile [14], Streptococcus pneumoniae [15], and Campylobacter [16]. Moreover, research on infectious diseases and probiotics 
has recently been extended to antibiotic-resistant superbugs [17] such as vancomycin-resistant Enterococcus (VRE) [18], methicillin-resistant Staphylococcus aureus (MRSA) [19], carbapenem-resistant Enterobacteriaceae (CRE) [20], MDR Pseudomonas aeruginosa [21], and viral infections such as human immunodeficiency virus (HIV) [22] and severe acute respiratory syndrome coronavirus 2 (SARS-CoV-2) [23].

As mentioned above, there have been many reports of the preventive and therapeutic effects of probiotics on various infectious diseases. However, research on the effects of probiotics against tuberculosis is still lacking, and therefore we investigated probiotics with the goal of discovering potential anti-tuberculosis drug candidates. In this study, we report the anti-tuberculosis effect of Pediococcus acidilatici PMC202 isolated from traditional Korean fermented foods.

\section{Materials and Methods}

Isolation of Probiotic Strain from Traditional Fermented Foods

The probiotic strain was isolated from young radish kimchi, a Korean traditional fermented food, in the laboratory of Soonchunhyang University in 2018. The liquid portion of each sample was streaked onto a plate of MRS agar (de Man, Rogosa and Sharp, BD Difco, USA) using a loop. The plates were cultured in an aerobic incubator (general incubator, N-Biotek, Korea) at $37^{\circ} \mathrm{C}$. The colonies were then cultured in MRS broth (BD Difco) and stored at $-80^{\circ} \mathrm{C}$ in $15 \%$ glycerol stock. Identification was done by $16 \mathrm{~S}$ rRNA gene sequencing.

16S rRNA Gene Sequencing of the Probiotic Strain

The 16S rRNA gene sequencing was performed by Biofact (Korea). Briefly, DNA was extracted by repeated heating and ice-cooling. Primers 27F (5'-AGA GTT TGA TCC TGG CTC AG-3') and 1492R (5'-GGT TAC CTT GTT ACG ACT T-3') were used for PCR on a Hushrun PCR cycler (Biofact, Korea). The amplified PCR product was purified and sequenced using an ABI PRISM 3730XL DNA analyzer (Applied Biosystems, USA) using a BigDye Terminator v3.1 Cycle Sequencing Kit (Thermo Fisher Scientific, USA). Sequences were compared with the National Center for Biotechnology Information (NCBI) GenBank database using BLAST (basic local alignment search tool).

\section{Preparation of Probiotic Strain Extract}

All probiotic strains were cultured in $30 \mathrm{ml}$ of MRS broth (BD Difco) and incubated at $37^{\circ} \mathrm{C}$ in a shaking incubator (BioFree, Korea) for $24 \mathrm{~h}$. Cultures were adjusted to an $\mathrm{OD}_{600 \mathrm{~nm}}$ value of 1.0 using a spectrophotometer (DR 1900, Hatch, USA). After that, centrifugation was performed at 4,000 $g$ for $10 \mathrm{~min}$ using a centrifuge (Combi R515, Hanil Scientific, Inc., Korea), followed by washing with $0.85 \% \mathrm{NaCl}$ solution to remove medium components. This process was repeated three times to collect the pellet. After adding $1 \mathrm{ml}$ of $0.85 \% \mathrm{NaCl}$ solution to the pellet followed by vortexing, it was transferred to a Lysing Matrix B tube (MP Biomedicals, USA) containing $0.1 \mathrm{~mm}$ silica spheres and disrupted for $1 \mathrm{~min}$ using a homogenizer (FastPrep-24 5G, MP Biomedicals). The probiotic extract was then boiled for $10 \mathrm{~min}$ prior to anti-mycobacterial testing.

\section{M. tuberculosis Strain and Culture Conditions}

M. tuberculosis H37Rv (ATCC 27294) was purchased from the American Type Culture Collection (ATCC, USA), and XDR M. tuberculosis (KMRC 00203-00197) was obtained from the Korean Mycobacterium Resource Center (KMRC, Korea). Based on the microdilution technique, the concentrations of isoniazid (INH), rifampicin (RIF), streptomycin (STR), pyrazinamide (PZA), and ethambutol (EMB) were $0.19,0.09,0.19,>200$, and $0.78 \mu \mathrm{g} / \mathrm{ml}$ for M. tuberculosis H37Rv, respectively, and 25, 100, 1.56, >200, and $6.25 \mu \mathrm{g} / \mathrm{ml}$ for XDR M. tuberculosis, respectively [24]. M. tuberculosis was cultured at $37^{\circ} \mathrm{C}$ in Middlebrook $7 \mathrm{H} 9$ broth (BD Difco) supplemented with $10 \%$ OADC (oleic acid-albumin-dextrose-catalase) (BD Difco) and 0.5\% Tween 80 (Sigma-Aldrich, USA). All experiments using M. tuberculosis were conducted in an Animal Biosafety Level 3 Laboratory of Soonchunhyang University (ABSL-3, KDCA-20-3-04).

\section{Cell Line and Culture Conditions}

The murine macrophage RAW 264.7 cell line (KCLB 40071) was purchased from the Korean Cell Line Bank (KCLB, Korea). The cells were prepared in Dulbecco's Modified Eagle Medium (DMEM, Gibco) supplemented with $10 \%$ fetal bovine serum (FBS) (Gibco) and $1 \%$ antibiotics (100 U/ml penicillin and $100 \mu \mathrm{g} / \mathrm{ml}$ streptomycin) (HyClone, USA) followed by incubation at $37^{\circ} \mathrm{C}$ in a $5 \% \mathrm{CO}_{2}$ atmosphere.

\section{Intracellular Anti-Mycobacterial Activity Test with Ziehl-Neelsen Staining}

RAW 264.7 cells $\left(1 \times 10^{5}\right.$ cells $\left./ \mathrm{ml}\right)$ grown for $24 \mathrm{~h}$ in a $5 \% \mathrm{CO}_{2}$ incubator were seeded onto 2 -well cell culture slides (SPL Life Sciences, Korea) until confluence reached approximately 70-80\%. Cells were then exposed to H37Rv or XDR M. tuberculosis strains at a multiplicity of infection (MOI) of 10:1 for $2 \mathrm{~h}$ to induce intracellular infection. After washing the cells three times with $1 \times$ phosphate-buffered saline (PBS), $2 \mathrm{ml}$ of DMEM without antibiotics containing various concentrations of probiotic strain extract was added to each well and incubated for 3 days at $37^{\circ} \mathrm{C}$ with a $5 \% \mathrm{CO}_{2}$ atmosphere. Cells were then washed three times with $1 \times \mathrm{PBS}$ to remove residues. After Ziehl-Neelsen staining, the cells were observed with an optical microscope (AX10, Carl Zeiss, Germany) at $1,000 \times$ magnification.

Intracellular Anti-Mycobacterial Activity Test Using CFU Assay

The intracellular anti-mycobacterial activity test was similar to the test for intracellular anti-tuberculosis effect 
using Ziehl-Neelsen staining. In this test, 96-well plates were used. The volume of each well was $200 \mu \mathrm{l}$. The colony-forming unit (CFU) method was used instead of staining to measure the anti-mycobacterial effect. Other conditions such as cultured cell types, cell culture/density, and infection conditions were the same. After 3 days of incubation, the cells were lysed with distilled water (DW) on the principle of osmotic pressure. Dilutions (10-fold) were spread onto 7H10 agar medium (BD Difco) plates. The M. tuberculosis CFU counts were then determined one month later.

\section{Anti-Mycobacterial Activity in Coculture Conditions}

The in vitro anti-tuberculosis activity of the probiotic was tested by coculturing the probiotic strain $\left(2 \times 10^{6}\right.$ $\mathrm{CFU} / \mathrm{ml})$ and $M$. tuberculosis $\mathrm{H} 37 \mathrm{Rv}\left(2 \times 10^{8} \mathrm{CFU} / \mathrm{ml}\right)$. The broth medium used consisted of $10 \% \mathrm{MRS}$ broth and $90 \% 7 \mathrm{H} 9$ broth. Both strains were cultured for two weeks in an incubator at $37^{\circ} \mathrm{C}$ with shaking $(180 \mathrm{rpm})$. On days $0,3,6,9$, and 12 , the CFUs of $M$. tuberculosis were counted. At the same time, the acidity was measured using a pH meter. Conditions wherein the initial pH was adjusted to 5 or 6.8 using hydrochloric acid (Sigma-Aldrich) or sodium hydroxide (Sigma-Aldrich) were also analyzed.

\section{Cell Cytotoxicity}

To evaluate the cytotoxicity of the probiotic, trypan blue and methylene blue staining were performed. Briefly, RAW 264.7 cells were seeded onto 2 -well cell culture slides at a density of $1 \times 10^{5} \mathrm{cells} / \mathrm{ml}$ and then incubated at $37^{\circ} \mathrm{C}$ with a $5 \% \mathrm{CO}_{2}$ atmosphere for $24 \mathrm{~h}$ until confluency reached about $70-80 \%$. After incubation, cells were washed with $1 \times \mathrm{PBS}$ and then incubated at $37^{\circ} \mathrm{C}$ in a $5 \% \mathrm{CO}_{2}$ atmosphere for 3 days with probiotic extract. Cells were observed using an optical microscope (AX10, Carl Zeiss) after staining with methylene blue (Dagatron, Korea). The number of viable cells was measured with a hemocytometer (Marienfeld, Germany) after cells detached with a scraper were stained with trypan blue (Gibco, USA).

\section{Repeated-Dose Toxicity in Mice by Oral Administration}

Six-week-old female Balb/C mice were obtained from Koatech (Korea). Upon arrival, all animals were inspected for health status to confirm suitability for study. The mice were acclimatized to the laboratory environment for 7 days, housed (6 per cage) in an environment-controlled barrier animal room, and given free access to a standard commercial diet and drinking water ad libitum. All animal rooms were monitored and maintained under a 12 -h light/dark cycle $(150-300 \mathrm{Lux})$ at a temperature of $19-25^{\circ} \mathrm{C}$ and $30-70 \%$ relative humidity.

The probiotic strain was inoculated into MRS broth at $0.1 \%$, incubated at $37^{\circ} \mathrm{C}$ for $24 \mathrm{~h}$, and washed with $0.85 \%$ $\mathrm{NaCl}$ solution. The probiotic was then adjusted to $6 \times 10^{8} \mathrm{CFU} / \mathrm{ml}$, of which $200 \mu \mathrm{l}$ was orally administered once daily, five times a week, for a total of two weeks using a zonde. The control group was administered with a $0.85 \%$ $\mathrm{NaCl}$ solution without probiotics. Acute toxicity was assessed based on clinical signs, body weight, and mortality within the dosing period. At the end of the experiment, lungs were removed, and bronchoalveolar lavage (BAL) fluid was collected in the same way as previously reported [25].

This animal experiment was conducted at Soonchunhyang University's PMC Animal Lab, which is registered as an animal testing facility (KFDA 657) in accordance with the regulations of the Act on Laboratory Animals licensed as ABSL-2 (LML 20-591). The animal experimentation plan in this study was reviewed and approved by the Institutional Animal Care and Use Committee (IACUC) of Soonchunhyang University (Approval No. 20210047).

\section{Metagenomics Analysis of BAL Fluids}

According to the manufacturer's instructions, total DNAs were extracted from BAL samples using a QIAamp DNA Mini Kit (Qiagen). Next, the V4 hypervariable region was amplified using a primer set (Forward primer: TCGTCGGCAGCGTCAGATGTGTATAAGAGACAG-CCTACGGGNGGCWGCAG, Reverse primer: GTC TCGTGGGCTCGGAGATGTGTATAAGAGACAG-GACTACHVGGGTATCTAATCC) capable of amplifying the primer sequences $515 \mathrm{f}$ to $806 \mathrm{r}$ of the $16 \mathrm{~S}$ rRNA gene. The part before the dash in the primer was the overhang adapter sequence required later for indexing. The part after the dash in the primer was a locus-specific sequence to obtain a product of $359 \mathrm{bp}$ after the first PCR. Using a primary PCR product as a substrate, a metagenomic library was prepared using a Nextera XT DNA Library Prep Kit (Illumina, USA). For PCR, 2×KAPA HiFi HotStart ReadyMix (Kapa Biosystems, USA) was used. After each step, AMPure XP beads (Beckman Coulter, UK) were used for cleanup. The concentration and quality thereof were then checked. The sample was finally diluted from $1 \mathrm{nM}$ to $50 \mathrm{pM}$ with $10 \mathrm{mM}$ Tris (pH 8.5). After the addition of a $10 \%$ PhiX Control Library (Illumina), the library sample was loaded into an iSeq-100 reagent cartridge (Illumina). After sequencing with an iSeq-100 platform (Illumina), the sequencing data were analyzed using the EzBioCloud server (Cheonlab, Korea).

\section{Quantification of Nitric Oxide}

The concentration of nitrite $\left(\mathrm{NO}_{2}{ }^{-}\right)$, which is used as an indicator of nitric oxide $(\mathrm{NO})$ synthesis, was measured using Griess reagent as previously reported [26]. Briefly, RAW 264.7 cells were seeded into 96-well cell culture plates at a density of $1 \times 10^{5} \mathrm{cells} / \mathrm{ml}$ per well, cultured for $24 \mathrm{~h}$ at $37^{\circ} \mathrm{C}$, and then infected with M. tuberculosis. After washing three times with $1 \times \mathrm{PBS}$, the cells were treated with probiotic extract for 3 days. For NO quantification, $50 \mu \mathrm{l}$ of the cell culture supernatant was transferred to a new 96-well plate, mixed with the same amount of Griess reagent solution (G2930, Promega, USA), and incubated at room temperature for $10 \mathrm{~min}$. The absorbance was then measured at $540 \mathrm{~nm}$ with a microplate reader (Victor Nivo, Perkin-Elmer, USA). 
Whole-Genome Sequencing of Probiotic Strain

The probiotic strain was inoculated into MRS broth at a ratio of $0.1 \%$. Cells were obtained at the late exponential phase of growth. After washing three times with PBS, gDNA was extracted using a QIAamp DNA Mini Kit (Qiagen). PacBio library construction and whole-genome sequencing were performed by Chunlab. Genomic DNA was cut into $10 \mathrm{~kb}$ using a g-tube (Covaris, USA) and purified. Ends were repaired, and SMRTbell adapters were ligated to the blunt end using the SMRTbell Template Prep Kit 1.0 (PacBio, USA). The library was then sequenced using PacBio P6C4 chemistry in an 8-well-SMART Cell v3 of PacBio RSII (PacBio). PacBio sequencing data were assembled with PacBio SMRT Analysis 2.3.0 using the HGAP2 protocol. The genome was then circularized using a Circlator 1.4.0 (Sanger Institute, UK). Protein coding sequences (CDSs) were predicted with Prodigal 2.6.2 [27] and grouped according to roles regarding orthologous groups (EggNOG; http:// eggnogdb.embl.de). Genes encoding tRNAs were searched using tRNAscan-SE 1.3.1 [28]. rRNAs and other noncoding RNAs were searched by covariance model searches using the Rfam 12.0 database [29]. For comparison of prokaryotic genome sequences, OrthoANIu algorithm-based Average Nucleotide Identity (ANI) calculator (https://www.ezbiocloud.net/tools/ani) was used [30].

\section{Results}

16S rRNA Gene Sequencing-Based Identification of Isolated Probiotic

The probiotic strain isolated from young radish kimchi was taxonomically identified through 16S rRNA gene sequencing. This probiotic strain's $16 \mathrm{~S}$ rRNA gene sequence was compared with sequences deposited in the NCBI reference sequence database. The analysis result showed that the sequence of the strain was $99 \%$ similar to $16 \mathrm{~S}$ rRNA sequences of $P$. acidilactici strains DSM 20284 and NGRI 0510Q. In addition, the sequence was similar (97\% to $98 \%$ ) to those of strains P. pentosaceus DSM20336, P. stilesii FAIR-E 180, P. claussenii ATCC BAA-344, and $P$. argentinicus CRL 776, all belonging to genus Pediococcus (Table 1). These results indicate that the isolated strain could be a species in the genus Pediococcus.

\section{Whole-Genome Analysis Result of the Strain}

The whole-genome sequencing analysis result of the strain is shown in Fig. 1. The genome consists of 2,044,111 bp single circular chromosomes with 1,954 coding DNA sequences (CDSs) (Fig. 1A). A total of 1,929 proteins of predicted CDS were functionally classified according to the Clusters of Orthologous Groups (COGs) (Fig. 1B). Biological functions could be defined for 1,401 predicted proteins, while 528 CDS were homologous to conserved proteins with unknown functions in other organisms. The other 25 hypothetical proteins did not match with any known proteins in the database.

\section{OrthoANI Genomic Similarity}

Similarity analysis was performed using the OrthoANI method for strains that shared high similarities in the $16 \mathrm{~S}$ rRNA analysis using the entire genome sequence data of the strain (Fig. 2). The analysis result confirmed that its similarity with a strain (ZPA017, NGRI 0510Q) of P. acidilactici was $98.40 \%$. Its similarities with other species of the Pediococcus genus such as $P$. pentosaceus, $P$. claussenii, and P. damnosus were $74.77 \%, 70.13 \%$, and $70.13 \%$, respectively. As a result, it was confirmed that the newly discovered strain was a $P$. acidilactici species of the Pediococcus genus.

Comparison of Genomic Characteristics with Different Strains of P. acidilactici Species

The genome of PMC202 was then compared with published genome information on other strains of P. acidilactici (PMC48, K3, S1, JQII-5, HN9) (Table 2). The PMC 202 strain differed from other P. acidilactici strains in source, genome size, $\mathrm{G}+\mathrm{C}$ content, CDS, and rRNA/tRNA numbers. Thus, the PMC202 strain was judged as a new strain different from existing strains.

Table 1. 16S rRNA gene sequence analysis and blast analysis results with relevant data from NCBI.

\begin{tabular}{clcccc}
\hline NCBI reference & \multicolumn{1}{c}{ Organism } & Length & Score & Identities & Gaps \\
\hline NR_042057.1 & Pediococcus acidilactici DSM 20284 & 1569 & 2724 bits (1475) & $1496 / 1505(99 \%)$ & $5 / 1505(0 \%)$ \\
NR_041640.1 & Pediococcus acidilactici NGRI 0510Q & 1516 & 2695 bits $(1459)$ & $1467 / 1470(99 \%)$ & $3 / 1470(0 \%)$ \\
NR_042058.1 & Pediococcus pentosaceus strain DSM 20336 & 1569 & 2612 bits (1419) & $1478 / 1506(98 \%)$ & $5 / 1506(0 \%)$ \\
NR_042401.1 & Pediococcus stilesii strain FAIR-E 180 & 1529 & 2564 bits (1388) & $1460 / 1496(98 \%)$ & $3 / 1496(0 \%)$ \\
NR_075029.1 & Pediococcus claussenii strain ATCC BAA-344 & 1567 & 2510 bits (1359) & $1459 / 1507(97 \%)$ & $7 / 1507(0 \%)$ \\
NR_042623.1 & Pediococcus argentinicus strain CRL 776 & 1492 & 2477 bits (1341) & $1444 / 1494(97 \%)$ & $7 / 1494(0 \%)$ \\
NR_042232.1 & Pediococcus claussenii strain P06 & 1472 & 2418 bits $(1309)$ & $1418 / 1471(96 \%)$ & $6 / 1471(0 \%)$ \\
NR_029136.1 & Pediococcus parvulus strain S-182 & 1436 & 2302 bits $(1246)$ & $1374 / 1437(96 \%)$ & $6 / 1437(0 \%)$ \\
NR_043291.1 & Pediococcus ethanolidurans strain Z-9 & 1501 & 2344 bits $(1269)$ & $1404 / 1470(96 \%)$ & $6 / 1470(0 \%)$ \\
NR_113922.1 & Pediococcus parvulus strain NBRC 100673 & 1501 & 2381 bits $(1289)$ & $1428 / 1496(95 \%)$ & $8 / 1496(1 \%)$ \\
NR_043290.1 & Pediococcus cellicola strain Z-8 & 1542 & 2372 bits $(1284)$ & $1431 / 1503(95 \%)$ & $6 / 1503(0 \%)$ \\
NR_025388.1 & Pediococcus inopinatus strain DSM 20285 & 1551 & 2361 bits $(1278)$ & $1429 / 1503(95 \%)$ & $6 / 1503(0 \%)$ \\
NR_025388.1 & Pediococcus inopinatus strain DSM 20285 & 1551 & 2361 bits (1278) & $1429 / 1503(95 \%)$ & $6 / 1503(0 \%)$ \\
NR_042087.1 & Pediococcus damnosus strain DSM 20331 & 1561 & 2344 bits (1269) & $1427 / 1503(95 \%)$ & $11 / 1503(1 \%)$ \\
\hline
\end{tabular}

NCBI, National Center for Biotechnology Information. 
A

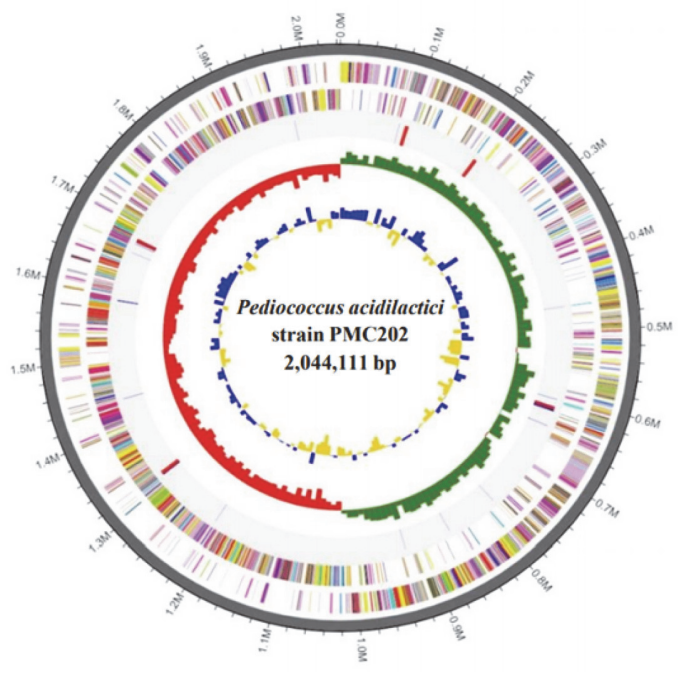

B $\mathrm{x}$

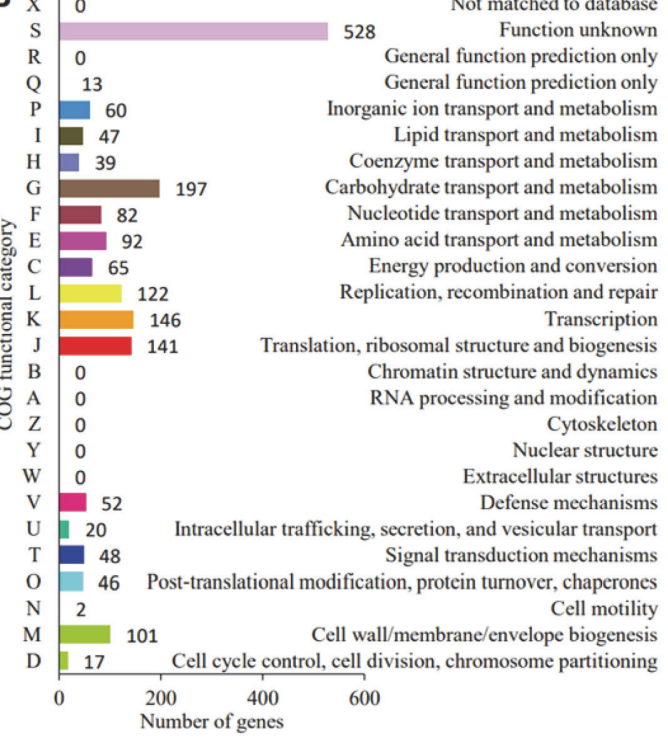

Fig. 1. High-throughput genome sequencing results of $P$. acidilactici strain PMC202. (A) The genome of the P. acidlactici PMC202 strain is shown as a circular map. Antisense/sense strands are colored according to the cluster of orthologous gene (COG) category. tRNA and rRNA are shown in red and blue from the periphery to the center. The inner-circle represents GC skew, with yellow and blue representing positive and negative values, respectively. GC content is shown in red and green. (B) Relative abundance by COG functional category cluster of genes is shown.
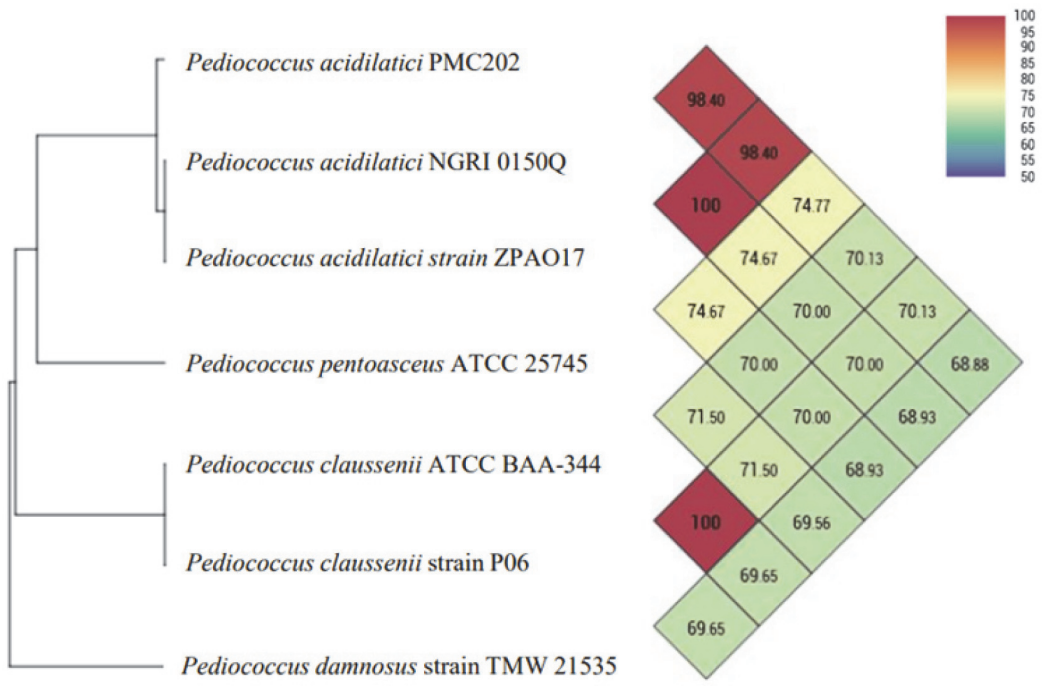

Fig. 2. OrthoANI results calculated from available genomes of Pediococcus species. The OrthoANI value of P. acidilatici PMC 202 and P. acidilatici NGRU $0150 \mathrm{Q}$ was 98.40 , higher than $96.0 \%$, the standard for determining the same species.

\section{Intracellular Anti-Mycobacterial Activity of PMC202}

The inhibitory effect of PMC202 on M. tuberculosis in macrophages was tested (Fig. 3). RAW 264.7 cells were infected with (A, B) M. tuberculosis $\mathrm{H} 37 \mathrm{Rv}$ or (C, D) XDR M. tuberculosis, treated with heat-treated PMC202 extract for 3 days, and analyzed by $(A, C) C F U$ method or (B, D) Ziehl-Neelsen staining method. Compared with the untreated control sample, PMC202 at $2.3 \times 10^{5} \mathrm{CFU} / \mathrm{ml}, 4.7 \times 10^{5} \mathrm{CFU} / \mathrm{ml}, 9.4 \times 10^{5} \mathrm{CFU} / \mathrm{ml}$, and $18.8 \times 10^{5}$ $\mathrm{CFU} / \mathrm{ml}$ significantly inhibited $M$. tuberculosis H37Rv. This effect was similar to INH at $1 \mu \mathrm{g} / \mathrm{ml}$ or $5 \mu \mathrm{g} / \mathrm{ml}$ (Fig. 3A). This anti-mycobacterial effect was also confirmed through staining. It was found that purple-colored M. tuberculosis increased at three days after infecting macrophages with M. tuberculosis (Fig. 3B). However, there was a relatively small amount of $M$. tuberculosis in samples treated with PMC202 or INH.

Unlike results for $M$. tuberculosis $\mathrm{H} 37 \mathrm{Rv}, 10 \mu \mathrm{g} / \mathrm{ml}$ of $\mathrm{INH}$ treatment had no significant anti-mycobacterial effect on XDR M. tuberculosis (Fig. 3C). However, the effect of PMC202 on XDR M. tuberculosis was similar to that on $M$. tuberculosis $\mathrm{H} 37 \mathrm{Rv}$ after treatment at $4.7 \times 10^{5} \mathrm{CFU} / \mathrm{ml}$ and $9.4 \times 10^{5} \mathrm{CFU} / \mathrm{ml}$. In particular, for samples 
Table 2. Comparison of chromosomal properties of $P$. acidilactici strains.

\begin{tabular}{lcccccc}
\hline \multicolumn{1}{c}{ Strain } & PMC202 & PMC48 & K3 & S1 & JQII-5 & HN9 \\
\hline Sources & $\begin{array}{c}\text { Young radish } \\
\text { kimchi }\end{array}$ & $\begin{array}{c}\text { Sesame leaf } \\
\text { kimchi }\end{array}$ & Nuruk & Makgeolli & Fermented dairy & Pork sausage \\
Genome size (bp) & $2,044,111$ & $2,043,929$ & $1,991,399$ & $1,980,172$ & $2,085,679$ & $2,107,472$ \\
G+C content (\%) & 42,2 & 42.2 & 42.1 & 42 & 42.2 & 42.1 \\
Predicted CDS & 1,954 & 2,026 & 1,525 & 1,525 & 1,840 & 1,990 \\
Number of rRNA genes & 15 & 15 & 8, & 7 & 15 & 15 \\
Number of tRNA genes & 57 & 57 & 50 & 40 & 57 & 56 \\
\hline
\end{tabular}

PMC202: https://www.ncbi.nlm.nih.gov/assembly/GCF_019448175.1 (PRJNA750221)

PMC48: https://www.ncbi.nlm.nih.gov/assembly/GCF_011604585.1

K3: https://www.ncbi.nlm.nih.gov/assembly/GCF_001294765.1

S1: https://www.ncbi.nlm.nih.gov/assembly/GCF_001461015.1

JQII-5: https://www.ncbi.nlm.nih.gov/assembly/GCF_006770685.1

HN9: https://www.ncbi.nlm.nih.gov/assembly/GCF_014906145.1

A

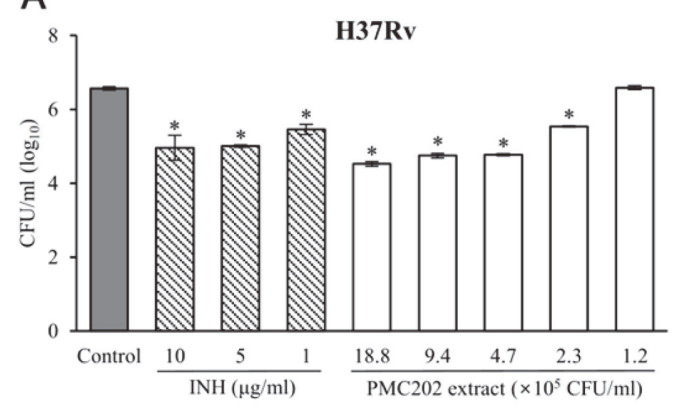

C

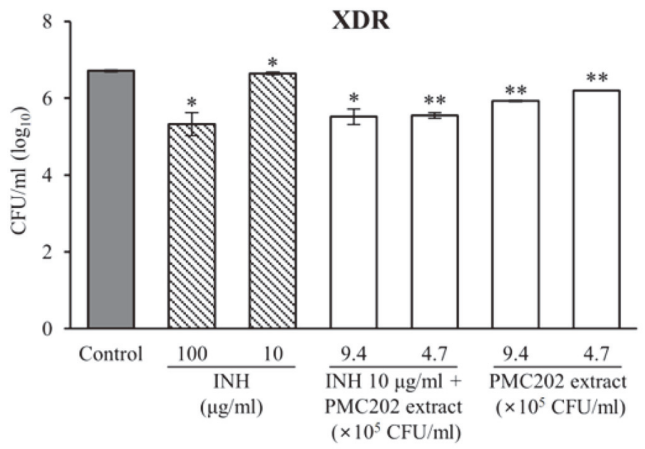

B

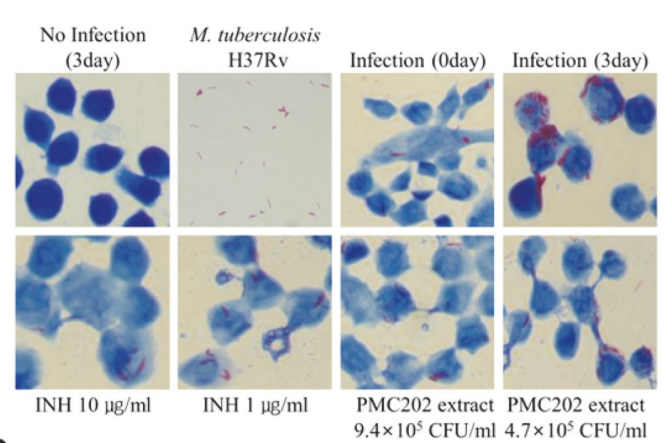

D

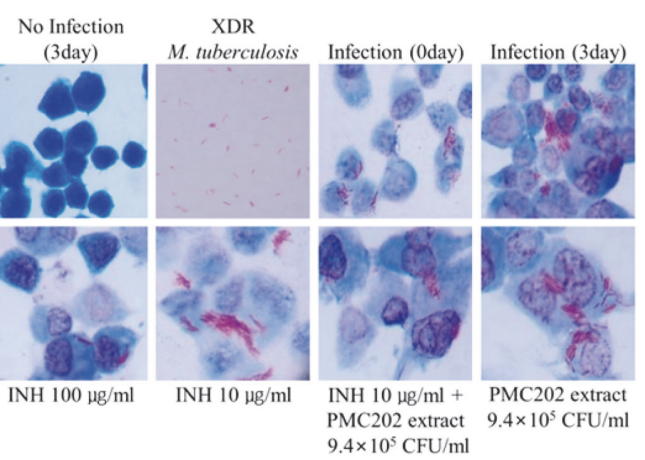

Fig 3. Effect of PMC202 on M. tuberculosis-infected macrophages. Macrophages were infected with (A, B) M. tuberculosis H37Rv or (C, D) XDR M. tuberculosis, treated with heat-treated PMC202 extract for 3 days. (A, C) CFU quantification results, (B, D) Ziehl-Neelsen staining images. The experiment was performed three times in triplicate. Values are expressed as mean values and standard deviations (SD). Statistical significance with controls was analyzed using unpaired Student's $t$-test. ${ }^{*} p<0.05 ;{ }^{* *} p<0.01$.

treated with $10 \mu \mathrm{g} / \mathrm{ml}$ of INH and $4.7 \times 10^{5} \mathrm{CFU} / \mathrm{ml}$ or $9.4 \times 10^{5} \mathrm{CFU} / \mathrm{ml}$ of PMC202 simultaneously, XDR M. tuberculosis was reduced more than that in samples treated with each alone. The anti-mycobacterial effect of PMC202 on XDR M. tuberculosis was also confirmed through the staining method (Fig. 3D).

\section{Anti-Tuberculosis Activity of PMC202 in Broth Coculture Condition}

The ability of PMC202 to inhibit M. tuberculosis H37Rv was evaluated in broth coculture conditions (Fig. 4). The CFU of M. tuberculosis (Fig. 4A) and the broth's pH (Fig. 4B) were measured on days 0, 3, 6, 9, and 12 while culturing $M$. tuberculosis alone or in a coculture with PMC202. The initial pH of M. tuberculosis single culture and coculture with PMC202 were 6.8 and 5.0, respectively, and after 12 days, the former increased to $8.1 \times 10^{8} \mathrm{CFU} / \mathrm{ml}$ and $\mathrm{pH} 7.0$, and the latter decreased to $8.7 \times 10^{4} \mathrm{CFU} / \mathrm{ml}$ and $\mathrm{pH} 4.5$. In addition, after 12 days of incubation, the culture of $M$. tuberculosis adjusted to the initial $\mathrm{pH}$ of 5 became $3.5 \times 10^{6} \mathrm{CFU} / \mathrm{ml}$ and $\mathrm{pH} 4.81$, and the coculture adjusted to the initial $\mathrm{pH}$ of 6.8 became $2.4 \times 10^{5} \mathrm{CFU} / \mathrm{ml}$ and $\mathrm{pH} 5.28$, and this decrease was greater than in the single culture. When PMC202 was cultured alone without $M$. tuberculosis, the $\mathrm{pH}$ gradually decreased during the incubation period and finally decreased to 4.3 . 
A

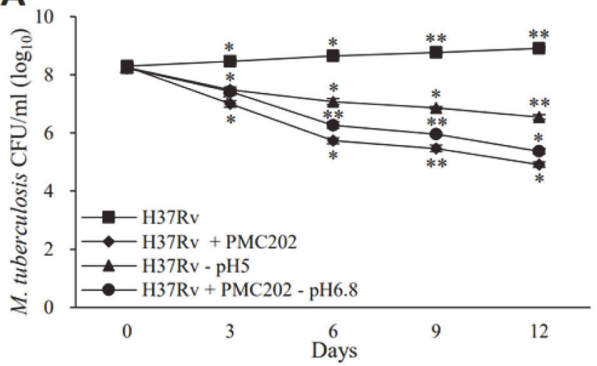

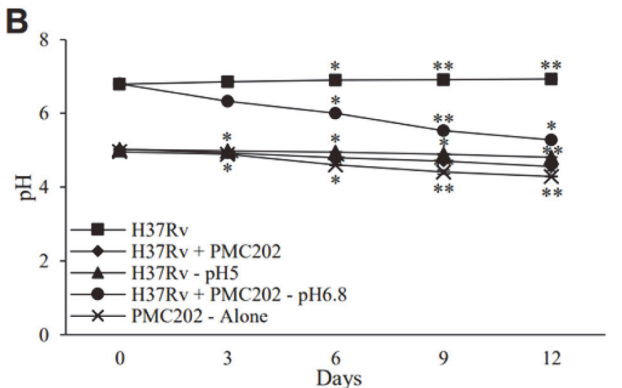

Fig. 4. Anti-mycobacterial activity of PMC202 against M. tuberculosis $\mathrm{H} 37 \mathrm{Rv}$ in coculture conditions. (A) The CFU of M. tuberculosis and (B) the $\mathrm{pH}$ of the culture were measured on days $0,3,6,9$, and 12 while culturing only M. tuberculosis (square) and coculturing M. tuberculosis and PMC202 (rhombus). The initial inoculation density was $2 \times 10^{8}$ $\mathrm{CFU} / \mathrm{ml}$ for M. tuberculosis, $2 \times 10^{6} \mathrm{CFU} / \mathrm{ml}$ for PMC202, and was cultured at $37^{\circ} \mathrm{C} 180 \mathrm{rpm}$ in $10 \mathrm{ml}$ of $7 \mathrm{H} 9$ broth containing $10 \%$ MRS broth. The initial pH of culturing $M$. tuberculosis alone and coculture conditions with $M$. tuberculosis and PMC202 were 6.8 and 5.0, respectively, and as time passed, both $\mathrm{CFU}$ and $\mathrm{pH}$ of $M$. tuberculosis increased in the former case and decreased in the latter case. In addition, the conditions of culturing only M. tuberculosis adjusted to an initial $\mathrm{pH}$ of 5.0 (triangle), coculture adjusted to an initial pH of 6.8 (circle), and culturing only PMC202 without M. tuberculosis (cross) were also measured. Experiments were performed three times in triplicate, and values are expressed as mean and SD. Statistical significance with initial value was analyzed using unpaired Student's $t$-test. ${ }^{*} p<0.05 ;{ }^{* *} p<0.01$.

\section{Cytotoxicity of PMC202}

The cytotoxicity of PMC202 extract to RAW 264.7 cells was evaluated (Fig. 5). The trypan blue staining test result showed that PMC202 at $9.4 \times 10^{5} \mathrm{CFU} / \mathrm{ml}$ or less did not affect the viability of macrophages. However, the viability of macrophages was significantly reduced when they were treated with PMC202 at a concentration higher than $18.8 \times 10^{5} \mathrm{CFU} / \mathrm{ml}$ (Fig. 5A). When cells were stained with methylene blue and observed under an optical microscope, cytotoxicity was observed when PMC202 at $18.8 \times 10^{5} \mathrm{CFU} / \mathrm{ml}$ or more was used for treatment, similar to the results of the trypan blue method (Fig. 5B).

\section{Repeated Oral Toxicity Assay of PMC202 in Mice}

Acute toxicity was investigated after mice were repeatedly treated with PMC202 through oral administration for two weeks (Fig. 6, Table 3). As a result, there was no significant change in body weight for mice administered with PMC202 compared to the group of mice administered with $0.85 \% \mathrm{NaCl}$ solution (Fig. 6). Death and unusual clinical changes were not observed after PMC202 administration (Table 3).

\section{Analysis of Microbiome Changes in BAL Fluid After Oral Administration of PMC202 to Mice}

The microbial community change in BAL fluid after administration of PMC202 was analyzed through a metagenomic analysis based on next-generation sequencing (NGS) technology (Fig. 7). After analyzing all of the applied statistical techniques, we confirmed that PMC202 administration did not cause a significant change in species richness (Fig. 7A) or diversity index (Fig. 7B). In the case of the averaged taxonomic composition at the phylum (Fig. 7C), class (Fig. 7D), or order (Fig. 7E) level, there was no significant difference among taxa having a composition of $1 \%$ or more. Moreover, beta-diversity analysis showed there was no significant difference in microbiome community between the two groups (Fig. 7F).

A

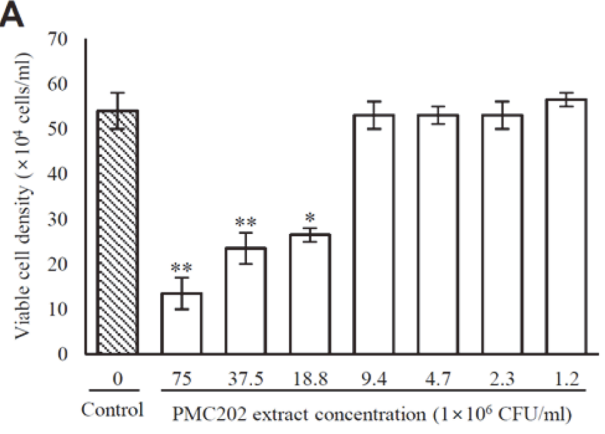

B

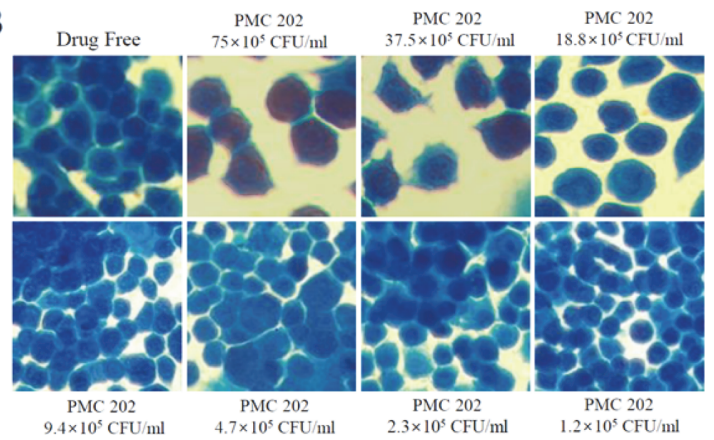

Fig. 5. Cytotoxicity evaluation of PMC202 using RAW 264.7 cells. RAW 264.7 cells were treated with heat-treated PMC202 extract at different concentrations for 3 days, (A) Live cells were counted with a hemocytometer after trypan blue staining, (B) Toxicity to cells was evaluated by methylene staining. The experiment was performed in triplicate three times. Values are expressed as mean \pm SD. Statistical significance vs. probiotics-free control was determined using unpaired Student's $t$-test. ${ }^{*} p<0.05 ;{ }^{* *} p<0.01$. 


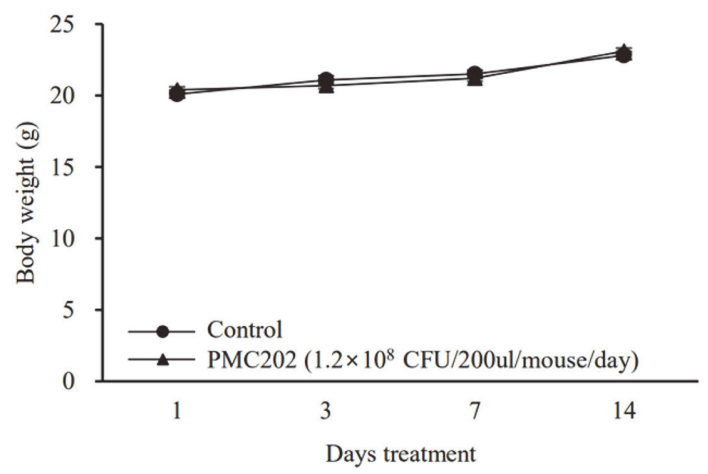

Fig. 6. Body weight of mice during two weeks of repeated oral administration of PMC202. All mice were weighed on days $1,3,7$, and 14 while the freshly prepared live PMC202 strain was orally administered at $1.2 \times 10^{8} \mathrm{CFU}$ per mouse once a day, five days a week, for two weeks.

Table 3. Clinical signs and mortality in a two-week oral toxicity study using mice.

\begin{tabular}{|c|c|c|c|c|c|c|c|c|c|c|c|c|c|c|c|c|}
\hline \multirow{2}{*}{ Group } & \multirow{2}{*}{$\begin{array}{c}\text { No. of } \\
\text { animals }\end{array}$} & \multirow{2}{*}{ Clinical sign } & \multicolumn{14}{|c|}{ Days after dosing } \\
\hline & & & 1 & 2 & 3 & 4 & 5 & 6 & 7 & 8 & 9 & 10 & 11 & 12 & 13 & 14 \\
\hline \multirow[t]{3}{*}{ Control } & \multirow[t]{3}{*}{6} & Loss weight (15\%) & 0 & 0 & 0 & 0 & 0 & 0 & 0 & 0 & 0 & 0 & 0 & 0 & 0 & 0 \\
\hline & & No observable abnormality & 0 & 0 & 0 & 0 & 0 & 0 & 0 & 0 & 0 & 0 & 0 & 0 & 0 & 0 \\
\hline & & Death & 0 & 0 & 0 & 0 & 0 & 0 & 0 & 0 & 0 & 0 & 0 & 0 & 0 & 0 \\
\hline \multirow{3}{*}{$\begin{array}{l}\text { PMC202 } \\
\left(1.2 \times 10^{8} \mathrm{CFU} /\right. \\
200 \mu \mathrm{l} / \mathrm{mouse} / \text { day })\end{array}$} & \multirow[t]{3}{*}{6} & Loss weight (15\%) & 0 & 0 & 0 & 0 & 0 & 0 & 0 & 0 & 0 & 0 & 0 & 0 & 0 & 0 \\
\hline & & No observable abnormality & 0 & 0 & 0 & 0 & 0 & 0 & 0 & 0 & 0 & 0 & 0 & 0 & 0 & 0 \\
\hline & & Death & 0 & 0 & 0 & 0 & 0 & 0 & 0 & 0 & 0 & 0 & 0 & 0 & 0 & 0 \\
\hline
\end{tabular}

PMC202, Freshly cultured live PMC202 strain not subjected to heat or mechanical lysis.

\section{Evaluation of the Effect of PMC202 on NO Production}

The effect of PMC202 on the production of NO was tested (Fig. 8). RAW 264.7 cells were infected with M. tuberculosis $\mathrm{H} 37 \mathrm{Rv}$ (Fig. 8) and treated with PMC202 for 3 days. Griess reagent for quantifying nitrite as a NO indicator was then used. Nitrite production was induced in RAW 264.7 cells at 3 days after infection with M. tuberculosis. PMC202 at $4.7 \times 10^{5} \mathrm{CFU} / \mathrm{ml}, 9.4 \times 10^{5} \mathrm{CFU} / \mathrm{ml}$, and $18.8 \times 10^{5} \mathrm{CFU} / \mathrm{ml}$ reduced nitrite production by $12.1 \%, 15.2 \%$, and $18.3 \%$ in M. tuberculosis $\mathrm{H} 37 \mathrm{Rv}$-infected macrophages.

\section{Discussion}

From mono-drug-resistant to MDR, XDR, and most recently TDR, the rapid evolution of $M$. tuberculosis will continue to make tuberculosis an even more incurable disease unless new treatment options are soon available [31]. To manage drug-resistant tuberculosis, a variety of potential strategies are being proposed, including the use of a pathogen-centric approach of developing new compounds with different mechanisms of action, repurposing drugs, using new analogues of existing anti-tuberculosis drugs, and using host-centric approaches of immunomodulators, therapeutic vaccines, immunity, and cell therapy [32]. As a form of alternative treatment, probiotics have recently been highlighted for their potential roles in controlling tuberculosis [33]. In this study, probiotics were applied to develop an alternative approach to solve the problem of antibiotic-resistant $M$. tuberculosis, and we have reported the anti-tuberculosis effect of $P$. acidilactici PMC202 isolated from Korean traditional fermented food.

PMC202, a bacterium isolated from young radish kimchi, was judged to be P. acidilactici according to similarity cutoff criteria of $98.65 \%$ based on $16 \mathrm{~S}$ gene sequencing [34] and $95 \%$ based on the whole genome [35]. In addition, PMC202 was determined to be a novel strain because its source and genetic characteristics were different from other strains of the P. acidilactici species.

PMC202 showed a significant anti-mycobacterial effect in the coculture experiment with $M$. tuberculosis H37Rv. This result was similar to the result of Lactobacillus reducing the number of M. bovis in coculture conditions. This effect was related to the $\mathrm{pH}$ decrease due to the organic acid production of lactic acid bacteria. Thus, despite its limitations as a pulmonary tuberculosis model, it might be suitable as an in vitro model of intestinal tuberculosis, which is known to account for 3 to $5 \%$ of extrapulmonary tuberculosis cases [36].

Tuberculosis infection of the host begins after inhalation of an aerosol containing a small number of bacilli [37]. Once entering the lungs, these bacilli are internalized through phagocytosis by alveolar macrophages [37]. RAW 264.7 macrophages are used as a general cell model in tuberculosis research [38], and were therefore used as an in vitro model in the present study. The intracellular anti-mycobacterial effect of PMC202 was then investigated. PMC202 showed an effect at a concentration that did not show cytotoxicity against drug-sensitive and -resistant M. tuberculosis. In particular, it also showed a synergistic effect with INH against XDR M. tuberculosis. These 
A

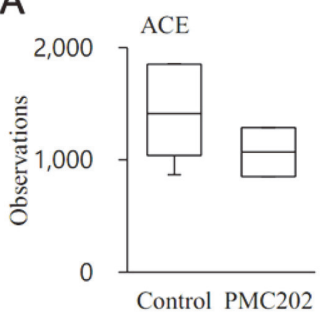

B

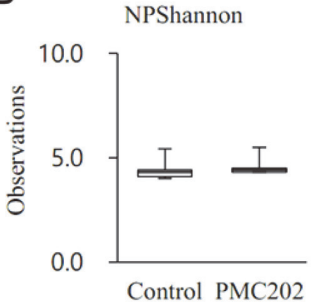

C

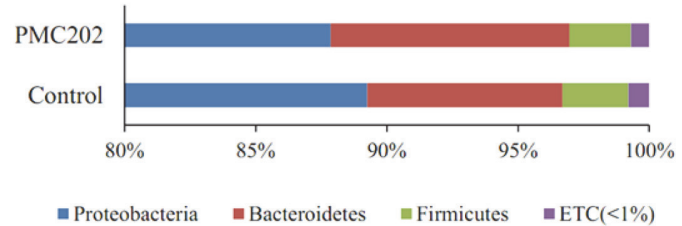

E
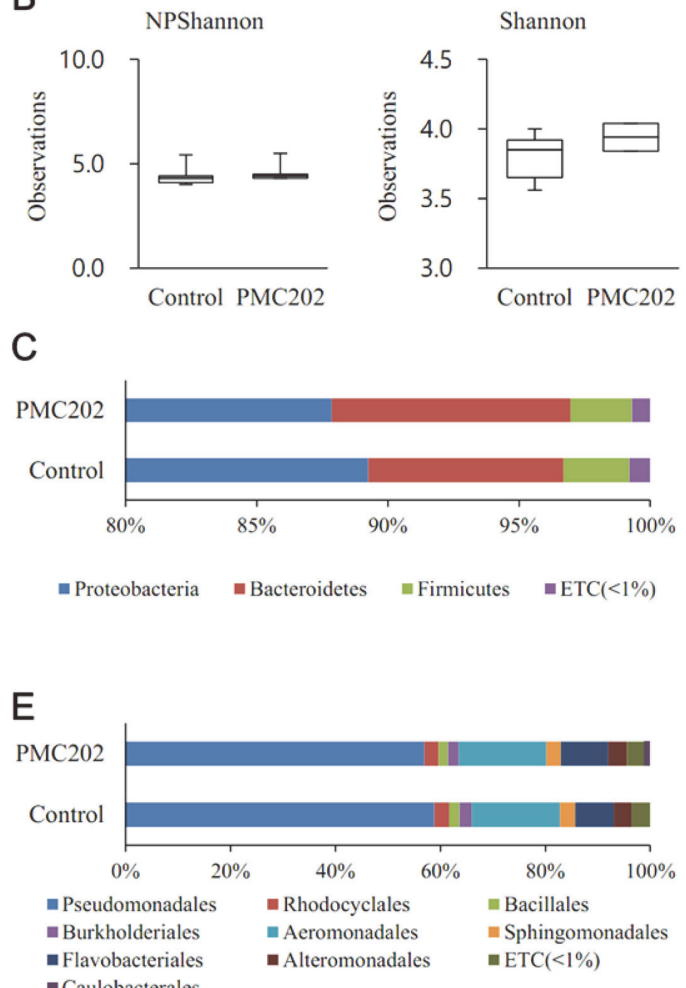
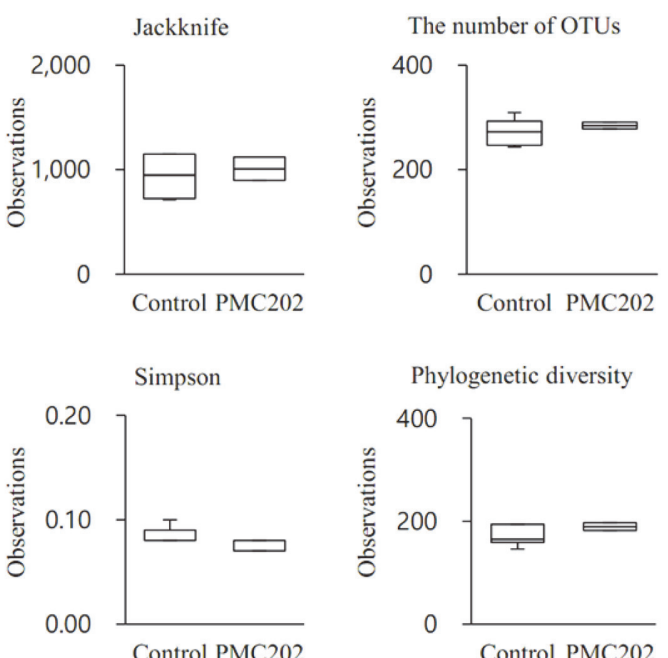

Phylogenetic diversity

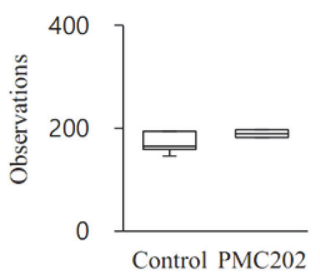

D

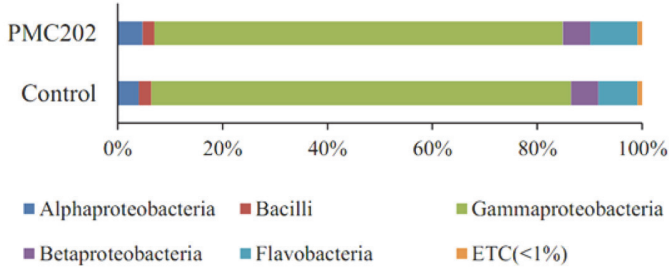

$\mathrm{F}$

\begin{tabular}{cc}
\hline Pair-wise & PERMANOVA results \\
\hline Jensen-Shannon & N.S. $(\mathrm{p}=0.105)$ \\
\hline Bray-Curtis & N.S. $(\mathrm{p}=0.598)$ \\
\hline Generalized UniFrac & N.S. $(\mathrm{p}=0.773)$ \\
\hline UniFrac & N.S. $(\mathrm{p}=0.437)$ \\
\hline
\end{tabular}

Fig. 7. Analysis of effects of PMC202 on lung microbiome in mice. Alpha-diversity was measured through (A) species richness and (B) diversity index. Averaged taxonomic compositions between two groups were compared at (C) phylum, (D) class, or (E) order level. (F) Beta-diversity was evaluated through beta set-significance analysis. Taxonomic relative abundance was analyzed using the In Wilcoxon rank-sum test. N.S., Not significant.

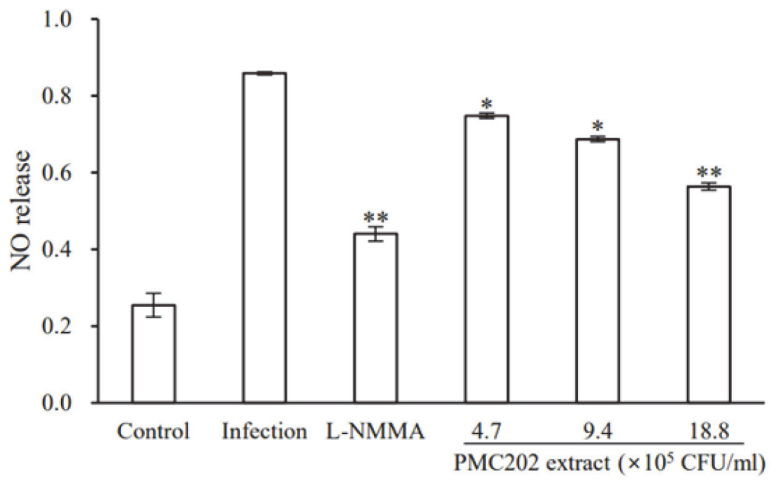

Fig. 8. Effects of PMC202 on nitrites $\left(\mathrm{NO}_{2}{ }^{-}\right)$in M. tuberculosis-infected macrophages. After infecting RAW 264.7 cells with M. tuberculosis H37Rv, heat-treated PMC202 extract was used for treatment for three days. As a nitric oxide (NO) indicator, nitrite was quantified using a Griess reagent. L-NG-mono-methylarginine (L-NMMA) was used as a negative control. Experiments were performed three times in triplicate. Values are expressed as mean \pm SD. Statistical significance vs. probiotic-free controls was determined using unpaired Student's $t$-test. ${ }^{*} p<0.05 ;{ }^{* *} p<0.01$. 
results indicate that PMC202 can be used as an adjuvant in conjunction with standard chemotherapy to treat M. tuberculosis infection.

Although currently available drugs are effective in treating tuberculosis disease or latent infection, they can cause serious side effects [39]. Drug-resistant tuberculosis, in particular, is treated with therapies that include second-line drugs with relatively high side effects, even death [40]. Therefore, toxicity evaluation is very important for newly developed anti-tuberculosis drugs. In this study, P. acidilactici was developed as an antituberculosis drug candidate, is generally recognized as safe (GRAS), and has probiotic properties such as beneficial enzyme activity [41]. Thus, it is widely used in the fermentation of food and starter culture for cheese and yogurt production [42].

Moreover, this strain was isolated from traditional fermented foods and is considered to be relatively safe. Despite this, there have been reports of toxicity and sepsis, especially in immunocompromised patients, even for strains well known as probiotics [43]. Therefore, a repeated oral administration toxicity test was conducted using mice for two weeks in this study. As a result, weight change, death, and specific clinical symptoms were not observed.

The microbiota that inhabits the body can modulate several endocrinal, neuronal, and immune pathways in the host, thus affecting essential human functions, including digestion, energy metabolism, and inflammation [44]. Antibiotic treatment can cause changes in the microbiome, depending on the type of antibiotic, dose, and duration of exposure. This dysbiosis is closely related to disease and health [45]. The WHO guidelines recommend 6 months of multi-drug therapy for new pulmonary tuberculosis patients [46]. However, patients with MDR-tuberculosis require high-dose chemotherapy with a second-line drug for 9 to 24 months [47]. There is a growing interest in the relationship between tuberculosis chemotherapy, which requires a high-dose combined antibiotic therapy over a long period of induction known to destroy the human microbiome and its side effects [48]. In recent years, the profound impact of anti-tuberculosis therapy on the composition of the lung microbiome, which plays a role in pathophysiological processes associated with tuberculosis disease, has become increasingly important [49]. Therefore, a metagenomic analysis based on the $16 \mathrm{~S}$ rRNA gene was performed for mouse BAL samples to evaluate the effect of oral administration of PMC202 on changes in the lung microbiome. As a result, PMC202 did not significantly affect species richness, species diversity, or taxonomic composition. It did not induce significant differences in microbial communities either.

Probiotics can regulate the innate/acquired immune system by influencing the mucosal/systemic immune response; thus, they are applied as immunotherapy [50]. From this perspective, the importance of the potential role of probiotics in the treatment of tuberculosis has been highlighted [33]. As such, the inhibitory effect of PMC202 on M. tuberculosis in macrophages seems to be related to the regulation of immune response. Therefore, we analyzed its association with NO, which is known to play a versatile role in the immune system [51]. The analysis showed that NO levels increased by M. tuberculosis infection were decreased by PMC202. This phenomenon can be interpreted several ways. As previously reported, it seems to be related to the cytoprotective effect [52]. Probiotics have been proposed to mediate immune responses by activating several inflammatory cytokines and interleukins associated with tuberculosis, but considering the lack of sufficient research, further studies are needed to elucidate the relevant mechanisms [33, 53].

In summary, this study showed the effects of $P$. acidlactici PMC202 newly isolated from young radish kimchi on M. tuberculosis in macrophages and suggested that it could be used as a candidate anti-tuberculosis agent for treating drug-resistant tuberculosis. However, more extensive studies, including evaluation of the in vivo animal efficacy of PMC202, clinical trials, and its mechanism of action, are needed. These findings highlight the potential role of using probiotics as a novel strategy in the treatment of tuberculosis.

\section{Acknowledgments}

This study was funded by the Ministry of Trade, Industry and Energy (MOTIE), Korea, under the 'Regional Industry-Based Organization Support Program' (reference number P0001942) supervised by the Korea Institute for Advancement of Technology (KIAT). This research was also supported by Soonchunhyang University Research Fund.

\section{Conflict of Interest}

The authors have no financial conflicts of interest to declare.

\section{References}

1. Orgeur M, Brosch R. 2018. Evolution of virulence in the Mycobacterium tuberculosis complex. Curr. Opin. Microbiol. 41: 68-75.

2. Koch A, Mizrahi V. 2018. Mycobacterium tuberculosis. Trends Microbiol. 26: 555-556.

3. Tarasuntisuk S, Palaga T, Kageyama H, Waditee-Sirisattha R. 2019. Mycosporine-2-glycine exerts anti-inflammatory and antioxidant effects in lipopolysaccharide (LPS)-stimulated RAW 264.7 macrophages. Arch. Biochem. Biophys. 662: 33-39.

4. Khawbung JL, Nath D, Chakraborty S. 2021. Drug resistant Tuberculosis: a review. Comp. Immunol. Microbiol. Infect. Dis. 74: 101574.

5. Chiang CY, Van Weezenbeek C, Mori T, Enarson DA. 2013. Challenges to the global control of tuberculosis. Respirology 18: 596-604.

6. Brust JCM, Shah NS, Mlisana K, Moodley P, Allana S, Campbell A, et al. 2018. Improved survival and cure rates with concurrent treatment for multidrug-resistant tuberculosis-human immunodeficiency virus coinfection in South Africa. Clin. Infect. Dis. 66: 1246-1253.

7. Reid G. 2016. Probiotics: definition, scope and mechanisms of action. Best Pract. Res. Clin. Gastroenterol. 30: 17-25.

8. Maldonado Galdeano C, Cazorla SI, Lemme Dumit JM, Velez E, Perdigon G. 2019. Beneficial effects of probiotic consumption on the immune system. Ann. Nutr. Metab. 74: 115-124.

9. Nomoto K. 2005. Prevention of infections by probiotics. J. Biosci. Bioeng. 100: 583-592. 
10. Goderska K, Agudo Pena S, Alarcon T. 2018. Helicobacter pylori treatment: antibiotics or probiotics. Appl. Microbiol. Biotechnol. 102: 1-7.

11. Gut AM, Vasiljevic T, Yeager T, Donkor ON. 2018. Salmonella infection - prevention and treatment by antibiotics and probiotic yeasts: a review. Microbiology (Reading) 164: 1327-1344.

12. Shenoy A, Gottlieb A. 2019. Probiotics for oral and vulvovaginal candidiasis: a review. Dermatol. Ther. 32: e12970.

13. Sihra N, Goodman A, Zakri R, Sahai A, Malde S. 2018. Nonantibiotic prevention and management of recurrent urinary tract infection. Nat. Rev. Urol. 15: 750-776.

14. Mills JP, Rao K, Young VB. 2018. Probiotics for prevention of Clostridium difficile infection. Curr. Opin. Gastroenterol. 34: 3-10.

15. Wong SS, Quan Toh Z, Dunne EM, Mulholland EK, Tang ML, Robins-Browne RM, et al. 2013. Inhibition of Streptococcus pneumoniae adherence to human epithelial cells in vitro by the probiotic Lactobacillus rhamnosus GG. BMC Res. Notes 6: 135.

16. Sorokulova IB, Kirik DL, Pinchuk II. 1997. Probiotics against Campylobacter pathogens. J. Travel Med. 4: 167-170.

17. Pamer EG. 2016. Resurrecting the intestinal microbiota to combat antibiotic-resistant pathogens. Science 352: 535-538.

18. Manley KJ, Fraenkel MB, Mayall BC, Power DA. 2007. Probiotic treatment of vancomycin-resistant enterococci: a randomised controlled trial. Med. J. Aust. 186: 454-457.

19. Karska-Wysocki B, Bazo M, Smoragiewicz W. 2010. Antibacterial activity of Lactobacillus acidophilus and Lactobacillus casei against methicillin-resistant Staphylococcus aureus (MRSA). Microbiol. Res. 165: 674-686.

20. Chen CC, Lai CC, Huang HL, Huang WY, Toh HS, Weng TC, et al. 2019. Antimicrobial activity of Lactobacillus species against carbapenem-resistant enterobacteriaceae. Front. Microbiol. 10: 789.

21. Nagasaki A, Takahashi H, Iinuma M, Uchiyama T, Watanabe S, Koide T, et al. 2010. Ulcerative colitis with multidrug-resistant Pseudomonas aeruginosa infection successfully treated with bifidobacterium. Digestion 81: 204-205.

22. Reikvam DH, Meyer-Myklestad MH, Troseid M, Stiksrud B. 2020. Probiotics to manage inflammation in HIV infection. Curr. Opin. Infect. Dis. 33: 34-43.

23. Manna S, Chowdhury T, Chakraborty R, Mandal SM. 2021. Probiotics-derived peptides and their immunomodulatory molecules can play a preventive role against viral diseases including COVID-19. Probiotics Antimicrb. Proteins 13: 611-623.

24. Kim S, Seo H, Mahmud HA, Islam MI, Kim YS, Lyu J, et al. 2017. In vitro effect of DFC-2 on mycolic acid biosynthesis in Mycobacterium tuberculosis. J. Microbiol. Biotechnol. 27: 1932-1941.

25. Van Hoecke L, Job ER, Saelens X, Roose K. 2017. Bronchoalveolar lavage of murine lungs to analyze inflammatory cell infiltration. J. Vis. Exp. 4: 55398

26. Zerin T, Lee M, Jang WS, Nam KW, Song HY. 2015. Ursolic acid reduces Mycobacterium tuberculosis-induced nitric oxide release in human alveolar A549 cells. Mol. Cells 38: 610-615.

27. Hyatt D, Chen GL, Locascio PF, Land ML, Larimer FW, Hauser LJ. 2010. Prodigal: prokaryotic gene recognition and translation initiation site identification. BMC Bioinformatics 11: 119.

28. Lowe TM, Eddy SR. 1997. tRNAscan-SE: A program for improved detection of transfer RNA genes in genomic sequence. Nucleic Acids Res. 25: 955-964.

29. Nawrocki EP, Burge SW, Bateman A, Daub J, Eberhardt RY, Eddy SR, et al. 2015. Rfam 12.0: updates to the RNA families database. Nucleic Acids Res. 43: D130-D137.

30. Yoon SH, Ha SM, Lim J, Kwon S, Chun J. 2017. A large-scale evaluation of algorithms to calculate average nucleotide identity. Antonie Van Leeuwenhoek 110: 1281-1286.

31. Nguyen L. 2016. Antibiotic resistance mechanisms in M. tuberculosis: an update. Arch. Toxicol. 90: 1585-1604.

32. Sharma D, Sharma S, Sharma J. 2020. Potential strategies for the management of drug-resistant tuberculosis. J. Glob. Antimicrob. Resist. 22: 210-214.

33. Liu Y, Wang J, Wu C. 2021. Microbiota and Tuberculosis: a potential role of probiotics, and postbiotics. Front. Nutr. 8: 626254

34. Mincheol Kim, JS Chun. 2014. Chapter 4 - 16S rRNA Gene-Based Identification of Bacteria and Archaea using the EzTaxon server. Methods Microbiol. 41: 61-74.

35. Aubin GG, Bemer P, Kambarev S, Patel NB, Lemenand O, Caillon J, et al. 2016. Propionibacterium namnetense sp. nov., isolated from a human bone infection. Int. J. Syst. Evol. Microbiol. 66: 3393-3399.

36. Chong VH, Lim KS. 2009. Gastrointestinal tuberculosis. Singapore Med. J. 50: 638-645; quiz 646.

37. Pieters J. 2008. Mycobacterium tuberculosis and the macrophage: maintaining a balance. Cell Host Microbe. 3: 399-407.

38. Pan FG, Zhao YY, Zhu S, Sun CJ, Lei LC, Feng X, et al. 2012. Different transcriptional profiles of RAW264.7 infected with Mycobacterium tuberculosis H37Rv and BCG identified via deep sequencing. PLoS One 7: e51988.

39. Forget EJ, Menzies D. 2006. Adverse reactions to first-line antituberculosis drugs. Expert Opin. Drug Saf. 5: 231-249.

40. Prasad R, Singh A, Gupta N. 2019. Adverse drug reactions in tuberculosis and management. Indian J. Tuberc. 66: 520-532.

41. Deen B, Diez-Gonzalez F. 2019. Assessment of Pediococcus acidilactici ATCC 8042 as potential Salmonella surrogate for thermal treatments of toasted oats cereal and peanut butter. Food Microbiol. 83: 187-192.

42. Bansal P, Kumar R, Singh J, Dhanda S. 2019. Next generation sequencing, biochemical characterization, metabolic pathway analysis of novel probiotic Pediococcus acidilactici NCDC 252 and it's evolutionary relationship with other lactic acid bacteria. Mol. Biol. Rep. 46: 5883-5895.

43. Redman MG, Ward EJ, Phillips RS. 2014. The efficacy and safety of probiotics in people with cancer: a systematic review. Ann. Oncol. 25: 1919-1929.

44. Lange K, Buerger M, Stallmach A, Bruns T. 2016. Effects of antibiotics on gut microbiota. Dig. Dis. 34: 260-268.

45. Iizumi T, Battaglia T, Ruiz V, Perez Perez GI. 2017. Gut microbiome and antibiotics. Arch. Med. Res. 48: 727-734

46. Romanowski K, Balshaw RF, Benedetti A, Campbell JR, Menzies D, Ahmad Khan F, et al. 2019. Predicting tuberculosis relapse in patients treated with the standard 6-month regimen: an individual patient data meta-analysis. Thorax 74: 291-297.

47. Lange C, Dheda K, Chesov D, Mandalakas AM, Udwadia Z, Horsburgh CR, Jr. 2019. Management of drug-resistant tuberculosis. Lancet 394: 953-966.

48. O'Toole RF, Gautam SS. 2018. The host microbiome and impact of tuberculosis chemotherapy. Tuberculosis (Edinb) 113: 26-29.

49. Hong BY, Maulen NP, Adami AJ, Granados H, Balcells ME, Cervantes J. 2016. Microbiome changes during tuberculosis and antituberculous therapy. Clin. Microbiol. Rev. 29: 915-926.

50. Eslami M, Bahar A, Keikha M, Karbalaei M, Kobyliak NM, Yousefi B. 2020. Probiotics function and modulation of the immune system in allergic diseases. Allergol. Immunopathol. (Madr). 48: 771-788.

51. Bogdan C. 2001. Nitric oxide and the immune response. Nat. Immunol. 2: 907-916.

52. Chakravortty D, Hensel M. 2003. Inducible nitric oxide synthase and control of intracellular bacterial pathogens. Microbes Infect. 5: 621-627.

53. Suprapti B, Suharjono S, Raising R, Yulistiani Y, Izzah Z, Nilamsari WP, et al. 2018. Effects of probiotics and Vitamin B supplementation on IFN-gamma and IL-12 levels during intensive phase treatment of tuberculosis. Indones J. Pharm. 29: 80-85. 